\title{
Proposal of an opportunistic approach for managing Mobility in Wireless community networks based on the Markov model
}

\author{
Askidi ABDOU ${ }^{\# 1}$, Thomas DJOTIO NDIÉ*2 Gilber NDJANPONG NANA \#3 \\ 1- LIRIMA, MASECNESS, University of Yaoundé I, National Polytechnic Higher School, \\ B.P 8390, Cameroon \\ 1 abdou_askidi@yahoo.fr; ${ }^{2}$ tdjotio@gmail; ${ }^{3}$ njanpong@yahoo.fr
}

\begin{abstract}
Today, there has been considerable progress in network and mobile radio technology. We are witnessing the deployment of different standards of mobile networks such as GSM, UMTS and wireless, such as WI-FI (IEEE802.11) and WIMAX (IEEE 802.16). Wireless community networks have grown rapidly with the emergence of Wi-Fi thanks to their simplicity, speed and low deployment cost. They are therefore an interesting alternative to conventional local networks. The management of mobility in wireless networks is now a challenge in this type of networks with the proliferation of mobile terminals. A mobile user can change his or her network access point by moving, without interrupting the current service session, that is, undergoing handover. Our study is located at the top layer of the OSI model (network, application and transport) from where we choose the SCTP / mSCTP protocols of the transport layer to support mobility thanks to multihoming with dynamic address reconfiguration RDA), the control of the borrowed addresses (or paths), the transfer of data in an association. And negligible losses of packets. We have modeled the mobility management using the Markov chain. Through the studies, on the functions of aggregations we also revealed the transmission rates which shows a better quality of service (QoS).
\end{abstract}

keywords: Community Wireless Networks (WSN), Mobility, the Markov chain, SCTP / mSCTP multihoming, functions of aggregations, QoS

\section{Introduction}

Within two decades, telecommunication systems have undergone profound changes and upheavals. Nowadays we have to do with the deployment of several networks using different technologies. These are telecommunications networks such as (GSM, UMTS) and wireless networks [(IEEE802.11 (WIFI) and IEEE 802.16 (WIMAX)] The deployment of GSM, UMTS and WIMAX networks is very costly compared to the IEEE 802.11x wireless network for its simple and fast implementation with a relatively low cost. Let us also note that in 1985, the U.S. Federal

Communications Commission (FCC) has decided to kept the bands reserved for industrial, scientific and medical use (ISM) between 902 and $928 \mathrm{MHz}, 2.4$ and $2.483 \mathrm{GHz}$, and 5.725 and $5.875 \mathrm{GHz}$, for unlicensed public use WLAN technologies [1]. We also note that [2] shows that many of the community networks have a wireless mesh architecture. Wireless mesh networks allow for rapid and simplified deployment, a high coverage development and, through their mesh, a high tolerance to breakdowns and interferences, significantly reducing the costs of installation and operation of networks. Figure 1 presents a mesh wireless architecture representing a scope (universities, schools .....).

In this paper, the focus was, in particular on mobility management, the guarantee of quality of service (QoS) in 802.11x community-based wireless networks. In a context of proximity, these types of networks are adequate and allow a user to connect anywhere: inside or outside (buildings). When moving from one cell to another, it is important that communications are stable (uninterrupted), reliable and transparent to ensure a better, if not acceptable level of QoS. the transport level mobility protocol mSCTP for advantages including multihoming is adopted. 


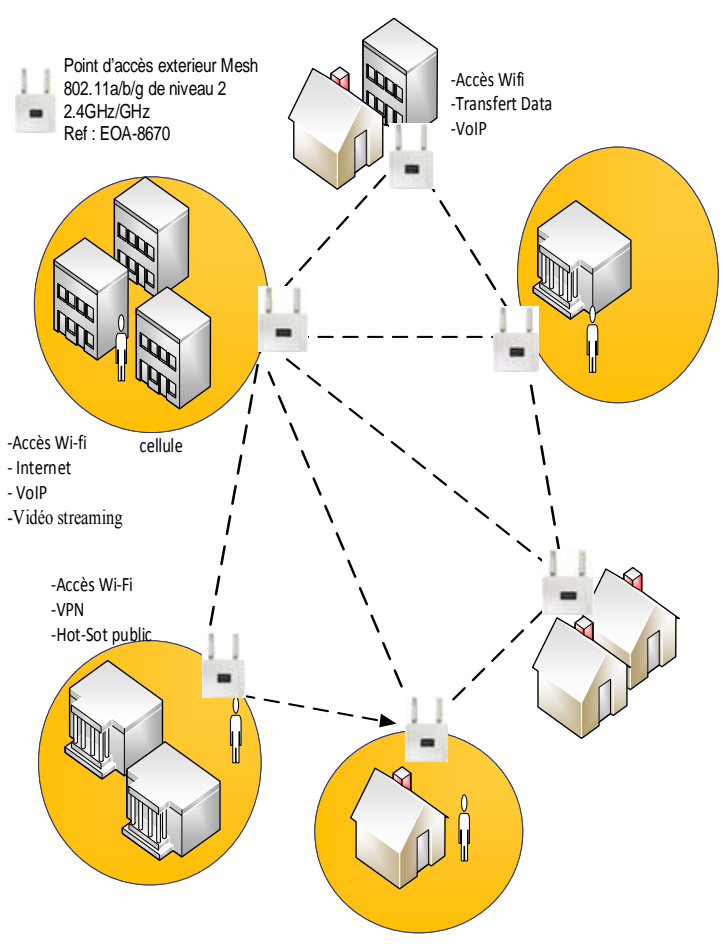

Figure 1:a mesh wireless architecture representing a scope (universities, schools .).

This community-based wireless network architecture includes: Wireless (WI-FI) access on all sites; The external access points Mesh 802.11a / b / g level 2 2.4GHz / 5GHz; Hot Spots for WiFi Internet access; The SSL VPN ensures security of access to internal network resources by using an SSL tunnel between the client (connected to a public network) and the VPN box.

This paper makes a choice on a mobility protocol that meets our context of managing mobility in wireless community networks. After studying the high layers of the OSI model (network, application and transport), the choice is made on the transport-level mSCTP protocol for advantages such as multihoming over network-level protocols (MIP) and application (SIP). The Markov chains to model the mSCTP protocol and the aggregation functions to show the audio quality of service (QoS) is used.

The next part of this article is presented as follow: the approach that is used to model the mSCTP mobility management protocol is described in part 2, and the results are shown in part 3. before concluding the work in part 5, a brief summary has to be done in part 4 .

\section{Methodology}

2.1 Protocols of mobility management: State of the art

In this section we present a synthesis of the most relevant mobility protocols in the upper layers of the OSI model: network, transport and application. the advantages and disadvantages of each protocol and justify our choice of the mSCTP protocol is presented.

\subsubsection{Mobility at network level: Protocol Mobile IP (MIP)}

Mobile IP (MIP) $[3,4]$ is presented as the current solution to the breakdown in communication problems during the movements of mobile nodes in IP networks. This protocol allows mobile nodes to move from networks to networks without breaking their ongoing sessions. The mobile node gets a new temporary IP address to each entry in a visited network, thanks to DHCP protocol and registers with its mother agent and the agent visited of the new network. This address indicates the current position of the mobile node. It will communicate with its home agent, which undertakes to intercept packets in the core network of the mobile node and to transmit it to its current position. Mobile IP can be divided into Mobile IPv4 (MIPv4) and Mobile IPv6 (MIPv6) depending on the version of the associated IP. Both protocols basically provide similar functionality with a few exceptions in the retail of operating mechanisms.

Although the Mobile IPv6 protocol allows to solve the triangular routing problem packet used in the Mobile IPv4 protocol, it still suffers from several weaknesses. These weaknesses, are: The time of handover is long. Specifically, the period of motion detection phase, the phase of the auto-configuration of addresses and of the association update phase are very long for real-time applications; the loss of Packet during the handover can be significant [5]. (HMIP, hierarchical MIP) (FMIP, fast handover for MIP) and F-HMIP are MIP improvements in 
order to reduce packet loss. However, none of these new solutions allows to have a low traffic signaling, falls within minimum and tolerable packet loss [3,6].

2.1.2 Mobility at the application level : SIP Protocol

SIP (Session Initiation Protocol) [3,4] is a signaling protocol of level defined by the IETF. It allows the establishment the release and the modification of multimedia sessions. It is based on a client / server model and proposes addressing SIP URL (Uniform Resource Locator) that resembles an email address. So a user of SIP protocol is reachable thanks to its SIP URL.

When the mobile moves into a new network or system, the SIP registration procedure is repeated to update the location. updates the location information will also be referenced by the proxy server (sometimes called a proxy) during initialization of session generated or terminated by the user agent (UA).

SIP protocol[4] base does not provide the transparent management of the handover. However, during a movement, the SIP protocol cannot guarantee the maintenance of a TCP/UDP session. However, the SIP protocol can be used in conjunction with other handoff management protocols such as Mobile IP (MIP); Cellular IP (CIP) or mSCTP (mobile Stream Control Transmission Protocol).

In the next section we present the SCTP / mSCTP Protocol upon which our choice is mode thanks to its multihoming function which is one of its peculiarities is the fact that several IP addresses can be activated within the same association. An error control mechanism to detect losses, rupture sequences or duplication of packets and dynamic configuration of addresses during an association [4].

\subsubsection{Transport Mobility: SCTP / mSCTP Protocol}

The Stream Command Transport Protocol (SCTP) [3,4] is a protocol an end-to-end, connection-oriented (or more precisely named association) that transports multiple streams of data. Multihoming and multistreaming $[3,5,11]$ missing to TCP allows SCTP endpoints to support multiple IP addresses (Figure 2). Multihoming protects an association from potential network failures by directing traffic to alternate IP addresses. During the initialization of an association, SCTP endpoints exchange IP address lists. Each endpoint can therefore send and receive messages from all IP addresses whose list is held by the endpoint. For example, one of the IP addresses listed will be designated as the primary address during initialization. If the primary address loses data packets repeatedly, all subsequent data packets will be transmitted to a replacement address until the primary address can be reestablished.

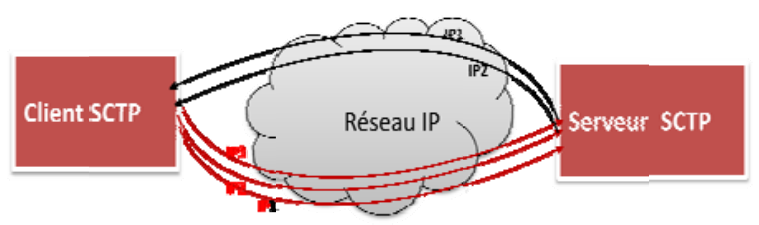

Figure 1: Example of SCTP Multihomed nodes.

\subsection{1 Managing IP addresses}

To transmit to a mulihomed node, the sender first chooses one of the possible addresses which corresponds to the primary path (when establishing the association). The transmitter must subsequently send data only via this primary path. In addition, in order to acknowledge SCTP packets from a multihomed node, the SACK chunks transmitter should use the same path as the chunks of received data [8]. However, when the receiver obtains multiple duplicate chunks, it may assume that its SACK chunks are using a failing path, so it may be wise to use another secondary address. When the primary path becomes inaccessible (due to congestion, or significant data loss), SCTP will switch all traffic to one of the secondary paths of the association in question.

2.1.3.2 Checking of borrowed addresses (or paths)

SCTP must regularly check the different addresses of a multihomed node. To do this, it considers two possible states for each of the possible paths: active or inactive. The primary path being considered active, the availability of the alternatives is controlled by the regular sending of HEARTBEAT Request chunks, which must be acknowledged by a HEARTBEAT ACK chunk. If an address does not respond after several unsuccessful HEARTBEATs, the path is considered inactive.

2. 1.3 3 Transferring data into an association with multihoming

As mentioned in [8], the use of multihoming in SCTP adds to the protocol the following basic functions:

- In an association, a single path is considered primary. This means that one of the IP addresses assigned to the receiver of the association is chosen to be the primary address.

-When acknowledging the received chunks, the SACK chunks must take the same route that was gone down by the received chunks. 
- In the case of retransmission of chunk towards a multihomed endpoint, the receiver must choose a destination address other than that to which the original data chunk was sent.

Thus the multihoming mechanism, supported by machines and network equipment, is a technically feasible and increasingly economic solution [9].

2. 1.3 .4 Multihoming and Mobility

Note that the multihoming character of SCTP makes it possible to support mobility over IP. Specifically, the SCTP protocol with dynamic address configuration extension can be used to provide smooth handover to terminals (MTs) that pass through different regions of IP networks during an activity session. This is called mobile SCTP (mSCTP) and applies to both IPv4 and IPv6 [4].

Mobility is managed by chunk: Address ConFiguration Change (ASCONF) and Address ConFiguration Ackowledgement (ASCONF-ACK) which offers the possibility to reconfigure IP addresses during the association; Changing the primary route (Routing to a new primary address); Exchanging layer adaptation information during association establishment. These addresses use parameters such as: Add IP Address (ASCONF): add a new IP address to the current association; Delete IP Address (ASCONF): allows to delete an IP address from the current association, etc.

2.2 Our contribution: modeling of the mSCTP protocol using the Markov chain.

We highlight the Markov chain to model the MSCTP protocol which constitutes for us the promoter protocol for the mobility management and the aggregation function to show the quality of service (QoS). We consider a cellular network composed of $\mathrm{n}$ cells and assume that these are all homogeneous and statistically identical.

2.2.1 Intuitive description model

$\mathrm{E}$ is the set of states of the system. $\mathrm{E}$ is over, and our system is composed of:

$\mathrm{C}=\left\{\mathrm{C}_{1}, \mathrm{C}_{2}, \ldots \ldots \ldots \mathrm{C}_{\mathrm{i}} \ldots \ldots . \mathrm{C}_{\mathbf{n}}\right\}=\{1,2, \ldots, \mathrm{i}, \ldots, \mathrm{n}\}$

represents the set of cells or states of the system environment.

$\mathbf{P}_{\mathrm{ij}}=\mathbf{P}\left(\mathbf{X}_{\mathrm{t}+1}=\mathbf{j} \mid \mathbf{X}_{\mathbf{t}}=\mathbf{i}\right)$

is the probability of transition from state $i$ to state $j$ during the time $t$ and $t+1$. This is a free system memory for the future state of the system depends only on its present state (this is the Markov property).

$\mathbf{P}_{\mathbf{t}}$ (i) is the probability that the mobile terminal is in the cell $\mathrm{C}_{\mathrm{i}}$ at the instant $\mathrm{t}$.

$\mathbf{m}$ is the number of connection (or more specifically named association in the SCTP terminology) between time $\mathrm{t}$ and $\mathrm{t}+1$.

$\operatorname{mSCTP}(\mathbf{t})$ is a subset of $\mathbf{C}$.

The matrix $\mathbf{M}_{\mathbf{t}+\mathbf{1}}=\left[\mathbf{P}_{\mathrm{ij}}\right]_{\mathbf{t}+\mathbf{1}}$ is a transition matrix (square matrix of order $\mathrm{n}$ which models the dynamics of the system transitions from time $t$ to time $t+1$ ). If for any time $t$,

$\mathrm{M}_{\mathrm{t}}=\mathrm{M}_{\mathrm{t}+1}$, then the system is called homogeneous (that is a special case of Markov management problems in practice).

2.2.2 Formal Model Description

$$
\begin{gathered}
P_{i j}=t[i, j] / g(i) \\
g(i)=\sum_{j=1}^{m} t(i, j)
\end{gathered}
$$

(2) is the number of transitions that have as a starting point the cell $\mathrm{i}$ during the $\mathrm{m}$ associations.

$\mathbf{t}[\mathbf{i}, \mathbf{j} \mathbf{j}$ est le nombre de transitions de la cellule i à la cellule $\mathbf{j}$ pendant les $\mathbf{m}$ associations avec le système.

$P_{t}(i)=k(i) / m$

with $\sum_{i=1}^{n} P_{t}(i)=1$ and $p_{i j} \geq 0$ for $\mathrm{i}, \mathrm{j} \in \mathrm{E}$. (Square matrix in which each element is a real between 0 and 1 and the sum of the elements of each row vaut1).

and $\sum_{i=1}^{n} K(i)=m$

$\mathbf{K}$ (i) is the number of times that it connects to the cell $\mathrm{Ci}$ in the $\mathrm{m}$ groups. 
$V=\left(P_{t}\left(C_{1}\right), P_{t}\left(C_{2}\right), P_{t}\left(C_{3}\right), \ldots ., P_{t}\left(C_{n}\right)\right)=\left(P_{t}(1), P_{t}(2), P_{t}(3), \ldots . ., P_{t}(n)\right)$

is the vector position probability at time $t$ of the user in the $n$ cells of the system.

$$
P_{t+1}(j)=\sum_{i=1}^{n} P_{t}(i) * P_{i j}
$$

is the probability that the mobile terminal is in the cell $\mathrm{Cj}$ at time $\mathrm{t}+1$. Consider a parameter $\delta$, with $\delta \in[0,1]$, the fixed or variable threshold to select the cells in large probability selected by the mSCTP protocol.

$\mathbf{m S C T P}=\left\{\mathrm{Cj} / \mathrm{P}_{\mathrm{t}+1}(\mathrm{j}) \geq \delta\right\}$

2.2.3 Network Modeling in graph form by the Markov chain

The transition matrix of a finite Markov chain can be associated with a graph whose vertices are the states. Pij are the state transition probabilities (i) to (j) (Figure 2).

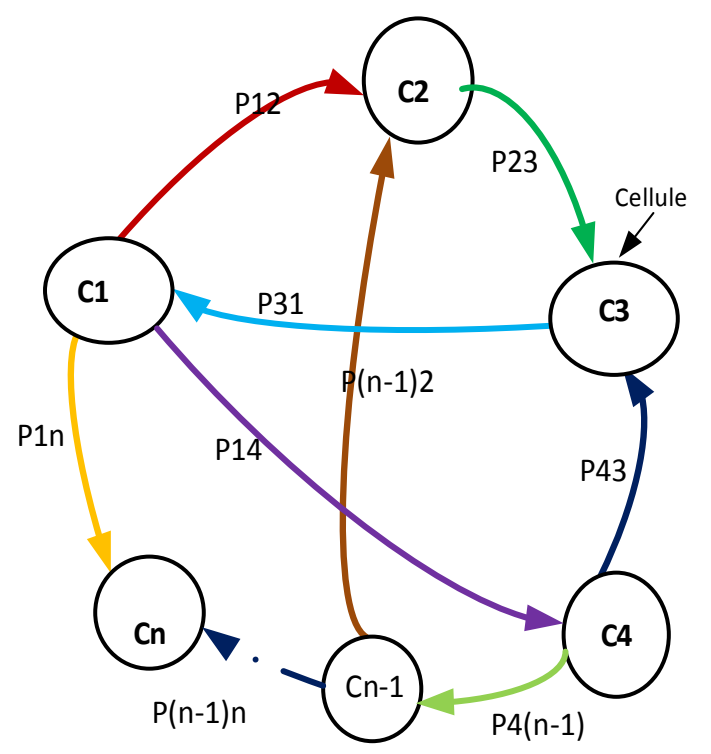

Figure 2: graph associated with a transition matrix between $\mathrm{n}$ states

\subsubsection{Aggregation functions for Quality of Service (QoS)}

Aggregate functions allow us to provide quantifiable judgment on several intercellular transitions that can guarantee the quality of service. To reach a consensus on these judgments, classical aggregation functions have been proposed: the arithmetic mean and almost arithmetic, geometric mean and almost geometric, median and many others $[10,12]$.

If $P_{i j}=P\left(X_{t+1}=j \mid X_{t}=i\right)$ is the probability of transition from state $i$ to state $j$ during the time $t$ and $\mathrm{t}+1$, For $\mathrm{n}=4$ (number of cells), the graph of the figure 3 we give:

$\mathrm{P} 11=0 ; \mathrm{P} 12=0.5 ; \mathrm{P} 22=0 ; \mathrm{P} 23=1 ; \mathrm{P} 31=1 ; \mathrm{P} 33=0 ; \mathrm{P} 43=1 ; \mathrm{P} 14=0.5 ; \mathrm{P} 44=0$.

Note also that $\mathrm{C} 1$ is the initial state, each state corresponds to an average of random time $(0,139 \mathrm{~s}, 0,138 \mathrm{~s}$, $0,137 \mathrm{~s}, 0,140 \mathrm{~s}$ ) for data type audio streaming[13]

$\mathrm{Ti}$ is the transmission rate at the $\mathrm{ith}$ associations $\Rightarrow \mathrm{Ti}=\mathrm{F}\left(\mathrm{T}_{1}, \mathrm{~T}_{2}, \mathrm{~T}_{3}, \ldots ., \mathrm{T}_{\mathrm{n}}\right) \in[0,1] . \mathrm{T}_{\mathrm{i}}$ represents the quality of service (QoS) and $\mathrm{F}$ the aggregation function.

$\mathrm{T}_{\mathrm{i}}=\frac{Q^{\prime} i+1}{Q i} \in[0,1] \Rightarrow \mathrm{Q}^{\prime}{ }_{\mathrm{i}+1} \leq \mathrm{Q}_{\mathrm{i}}$ is the quantity of information transmitted from $\mathrm{C}_{\mathrm{i}}$ to $\mathrm{C}_{\mathrm{i}+1}$. At this point transmission we assume that the user will receive any new information.

If $\mathbf{U}$ is the set of information being received in the new cell, then $Q_{i+1}=Q_{i+1}^{\prime}+\mathbf{U}$, with $Q_{i+1} \geq Q_{i+1}^{\prime}$ which is also the amount of information made available to the user at the input of the second transmission region (2nd Handover ) .

2.2.4. 1 Seek the aggregation function that meets our application context

$[10,12,16]$ show that the compromise function is located by definition between disjunctive and connective functions and fills most of the properties of the aggregation functions which shows the different transmissions that can be either unidirectional (simplex), Altered (half-duplex), bidirectional (full-duplex), ect. Hence it will be useful for us to better respond to the quality of service in our context. 


\subsubsection{Compromises operators}

Two average are entering the contribution of aggregate functions: the arithmetic mean (8) And the quasi-linear mean (9).

$$
\begin{aligned}
& F(X)=f^{-1}\left[\frac{1}{m} \sum_{i=1}^{m} f\left(x_{i}\right)\right] \\
& F(X)=f^{-1}\left[\frac{1}{m} \sum_{i=1}^{m} w_{i} f\left(x_{i}\right)\right]
\end{aligned}
$$

Among the two functions (8) and (9), the quasi-linear mean is distinguished by its weight wi (signal strength) which represents the weight of the handover and depends on the altitude between two interfering antennas, Obstacles while verifying the condition:

$$
\sum_{i=1}^{m} \mathcal{W}_{i}=1 \quad \text { avec } \quad \mathrm{w}_{\mathrm{i}} \in[0,1]
$$

\section{Results}

\subsection{Simulation results in Matlab}

Figure 3 a graph having 4 cells $(n=4)$ and 5 associations $(m=5)$. This result is to know in which cell the user is free to move at time $\mathrm{t}+1$ from the calculation:

$$
P_{t+1}(j)=\sum_{i=1}^{m} P_{t}(i) * P_{i j}
$$

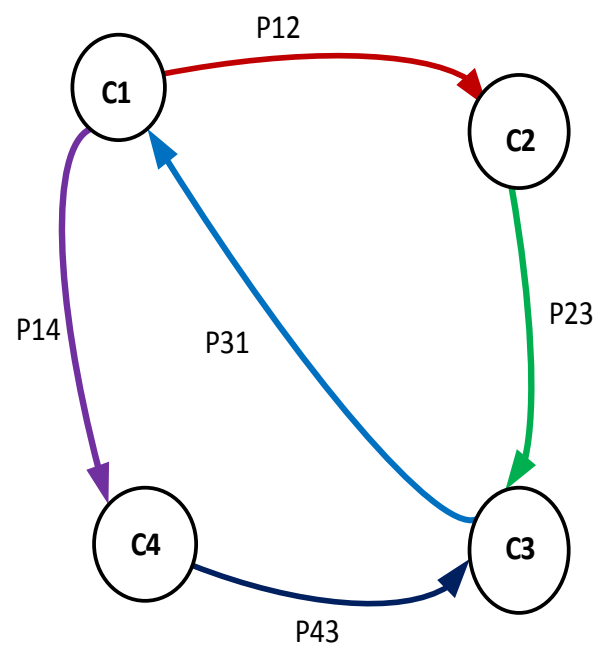

Figure 3: Representation graph of 4 cells and 5 associations.

Finally, the simulation carried out under a Matlab platform is written by the program below.

function [Cellules] = cellules $\operatorname{Markov}(\mathrm{T}$, Theta,tpn $)$

$\%$ Détermination des $\mathrm{g}(\mathrm{i})$

$\mathrm{g}=\operatorname{sum}\left(\mathrm{T}^{\prime}\right)$

\%Détermination des $\mathrm{K}(\mathrm{i})$

$\mathrm{K}=\operatorname{sum}(\mathrm{T})$;

\%Détermination des dimensions de $\mathrm{T}$; $\mathrm{n}$ :lignes, m:colonnes

$[\mathrm{n}, \mathrm{m}]=\operatorname{size}(\mathrm{T})$;

$\%$ Détermination du nombre d'association

nbAss $=\operatorname{sum}(K)$;

\%Détermination du vecteur de probabilité à l'instant

't' ("initiale") 
$\mathrm{Pt}=\mathrm{K} . / \mathrm{nbAss}$

\%Détermination de la matrice de trasition $\mathrm{P}$

for $\mathrm{i}=1: \mathrm{n}$

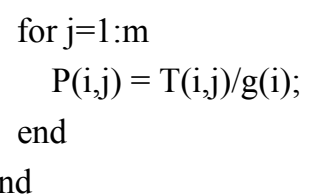

$\%$ Calcul des probalités de chaque cellule

Cell=Pt*P;

for $\mathrm{k}=2: \mathrm{tpn}$

Cell $=$ Cell $*$ P

end

\%Détermination des cellules vérifiants le critère "Theta" (les colonnes avec pour valeur "1")

for $\mathrm{j}=1: \mathrm{m}$

$$
\begin{aligned}
& \text { if Cell }(\mathrm{j})>=\text { Theta } \\
& \text { Cellules }(\mathrm{j})=1 ; \\
& \text { else } \\
& \text { Cellules }(\mathrm{j})=0 ; \\
& \text { end }
\end{aligned}
$$

end

Finally, the simulation carried out under a Matlab platform gives us.

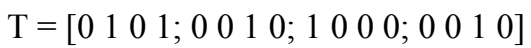

$\mathrm{T}=$

$\begin{array}{llll}0 & 1 & 0 & 1 \\ 0 & 0 & 1 & 0 \\ 1 & 0 & 0 & 0 \\ 0 & 0 & 1 & 0\end{array}$

$[\mathrm{C}]=$ Markov cells $(\mathrm{T}, 1 / 3,1)$

$\mathrm{P}=$

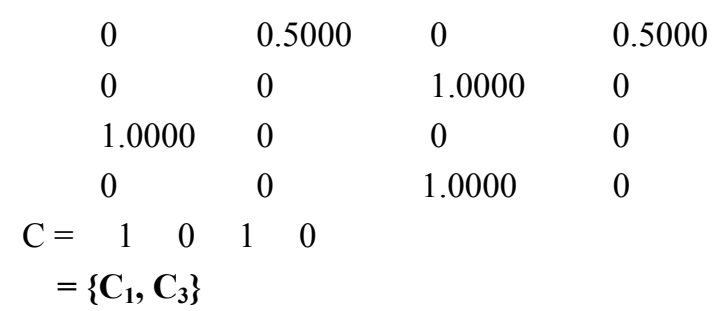

Matlab simulation allowed us to get if $\delta=\frac{1}{3}$ threshold is fixed, then MSCTP at time $\mathrm{t}+1$,

$\operatorname{mSCTP}(t+1)=\{\mathrm{Ci} / \mathrm{Pt}+\mathbf{1}(\mathbf{j}) \geq \delta\}=\{\mathrm{C} 1, \mathrm{C} 3\}$ If the selection criterion in all $\mathrm{mSCTP}(\mathrm{t}+\mathbf{1})$ is the cell having the highest probability, then the user will be switching between $\mathrm{C} 1$ and $\mathrm{C} 3$ inconsequential

3.2 Quality of service during a transfer intercellular

We propose four cases according to different levels [2] by varying the functions.

1st case: : Handover with a level(figure 4).

Measure the quality of service when $f$ is an identity function that is, $f(x)=x$ then

$T=F(X)=f^{-1}\left[\sum_{i=1}^{m} \mathcal{W}_{i} f\left(x_{i}\right)\right]=\sum_{i=1}^{m} \mathcal{W}_{i} x_{i}$

Let $Q_{i}=t_{i *} D_{i}$ ( ti is the random transmission time for each cell and Di throughput ) 
$T_{i}=\frac{Q_{i+1}^{\prime}}{Q_{i}} \quad$ with $\quad Q_{i+1}=Q_{i+1}^{\prime}+U$

$U$ is new information.

We take for example the audio conversion with $0,139 \mathrm{~s} ; 0,138 \mathrm{~s} ; 0,137 \mathrm{~s} ; 0,140 \mathrm{~s} ; 0,145 \mathrm{~s}$ random time allocated to each state and a constant rate of $40 \mathrm{kbit} / \mathrm{s}$ for all the cells $\mathrm{Ci}$.

We present first the simulation results as a function of $\mathrm{f}$ that is an identity function that is, $\mathrm{f}(\mathrm{x})=\mathrm{x}$, the weight $\mathrm{w}$ and quality of service T(Figure 5).

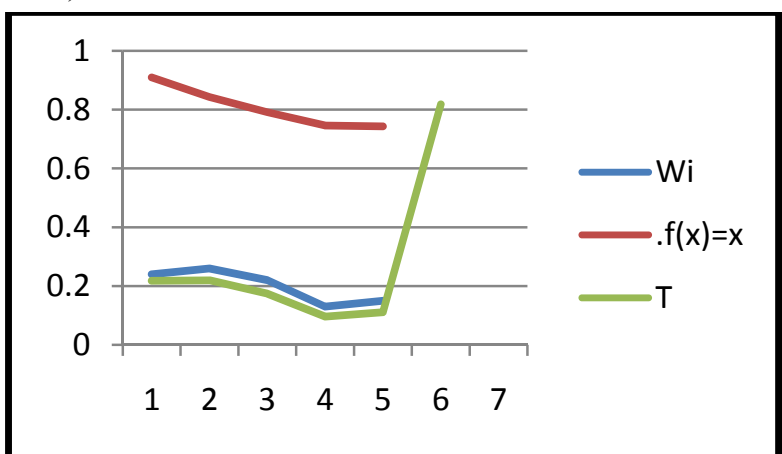

Figure 5: Quality of Service during a handoff with a level. Right here

$\mathrm{T}=0,82 \in[0,1]$.

2nd case: Handover with two levels(figure 6) level

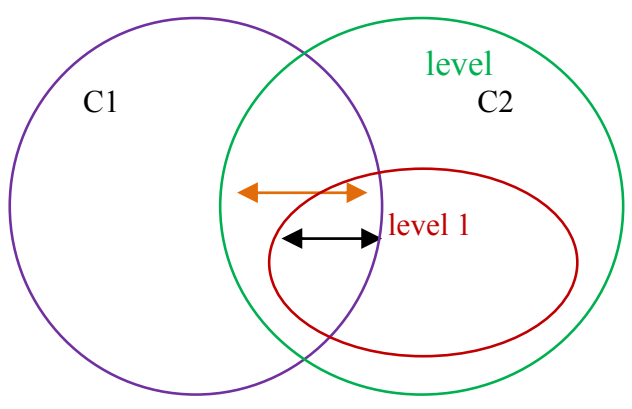

Figure 6: Handover with two levels

If Di is constant level by : $\mathrm{Q}_{22}=\mathrm{D}_{1} \mathrm{t}_{2}{ }_{2}+\mathrm{D}_{2} \mathrm{t}_{2}{ }_{2}$ with $\mathrm{t}=\mathrm{t}_{2}{ }^{\prime}+\mathrm{t}^{\prime \prime}{ }_{2}$ and $\mathrm{Q}_{1}$ known ( $5 \mathrm{kbits} / \mathrm{s}$ ) then $\mathrm{T}_{1}=\frac{Q^{\prime 2}}{Q 1}$. We always maintain the audio call data : $0,125 \mathrm{~s} ; 0,130 \mathrm{~s} ; 0,125 \mathrm{~s} ; 0,125 \mathrm{~s}$ for level 1 and $0,139 \mathrm{~s} ; 0,138 \mathrm{~s} ; 0,137 \mathrm{~s} ; 0,140 \mathrm{~s}$ ; 0,145s for Level $2 \mathrm{Q}_{\mathrm{i}+1}=\mathrm{Q}_{\mathrm{i}}^{\prime}+\mathrm{U}$

The following combinations are calculated according to figure 3

$\underline{\text { 1ere }^{\text {ere }} \text { association: }}$ Q1 $=5 \mathrm{kbits}, \mathrm{Q}^{\prime} 2=\mathrm{D} 1 * \mathrm{t} 2+\mathrm{D} 2 * \mathrm{t}^{\prime} 2$ with s $\mathrm{T} 1=\frac{Q^{\prime 2}}{Q 1} \underline{\mathbf{2}^{\mathrm{eme}}}: \mathrm{Q}^{\prime} 3=\mathrm{D}_{1} * \mathrm{t}_{3}^{\prime}+\mathrm{D}_{2} * \mathrm{t}_{3}{ }_{3}$ with $\mathrm{T}_{2}=\frac{Q^{\prime 3}}{Q^{\prime} 2} ; \underline{\mathbf{3}^{\mathrm{eme}}}$ $: \mathrm{Q}_{1}^{\prime}=\mathrm{D}_{1} * \mathrm{t}_{1}{ }_{1}+\mathrm{t}_{1}{ }_{1} \mathrm{D}_{2}$ with $\mathrm{T}_{3}=\frac{Q^{\prime} 1}{Q^{\prime} 3} ; \underline{4^{\text {eme }}}: \quad \mathrm{Q}_{4}^{\prime}=\mathrm{D}_{1} \mathrm{t}_{4}^{\prime}+\mathrm{D}_{2} * \mathrm{t}^{\prime \prime}{ }_{4}$ with s $\mathrm{T}^{4}=\frac{Q^{\prime} 4}{Q^{\prime} 1} ; \quad \underline{\mathbf{5}^{\mathrm{eme}}}: \mathrm{Q}_{3}=\mathrm{D}_{1} * \mathrm{t}_{3}{ }_{3}+\mathrm{D}_{2}{ }^{*} \mathrm{t}_{3} \quad$ with $\mathrm{T}_{5}=\frac{Q^{\prime} 3}{Q^{\prime} 4}$

We present simulation results as a function of $\mathrm{f}$ that is an identity function that is, $\mathrm{f}(\mathrm{x})=\mathrm{x}$, the weight $\mathrm{w}$ and quality of service $\mathrm{T}$ (Figure 7). 


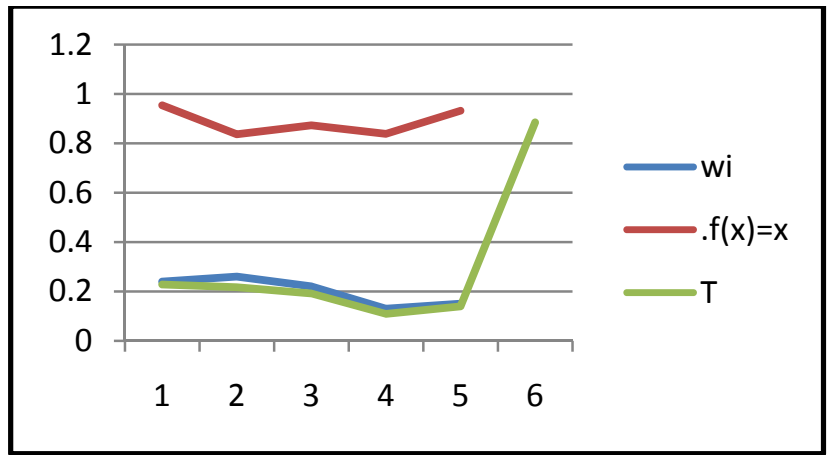

Figure 7: Quality of Service during a handoff between two levels.

$$
\mathrm{T}=0,892 \in[0,1]
$$

Resuming the calculation of the quality of service in the two previous cases, when $f(x)=\log x$ ie

$$
T=F(X)=f^{-1}\left[\sum_{i=1}^{m} w_{i} f\left(x_{i}\right)\right]=\prod_{i=1}^{m} x_{i}^{\mathcal{W}_{i}}
$$

$\mathbf{3}^{\text {eme }}$ cas: Handover with a level(Figure 8$)$.

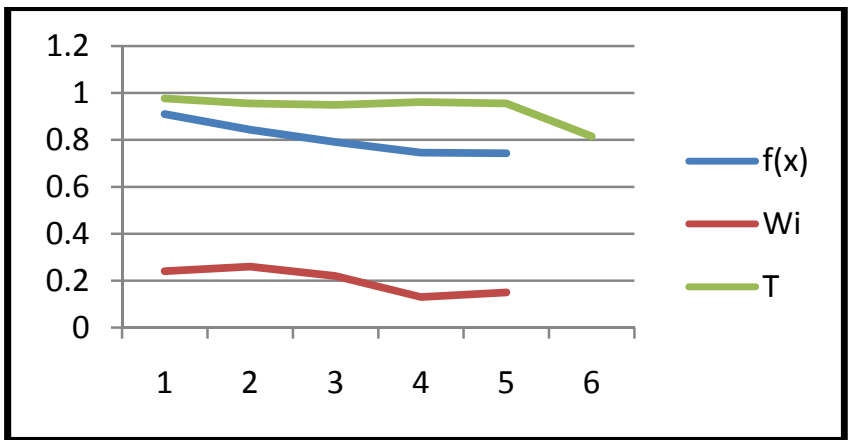

Figure 8: Quality of Service during a handover with a level.

$\mathrm{T}=0,815 \in[0,1]$

4th case : Handover with two levels(Figure 9).

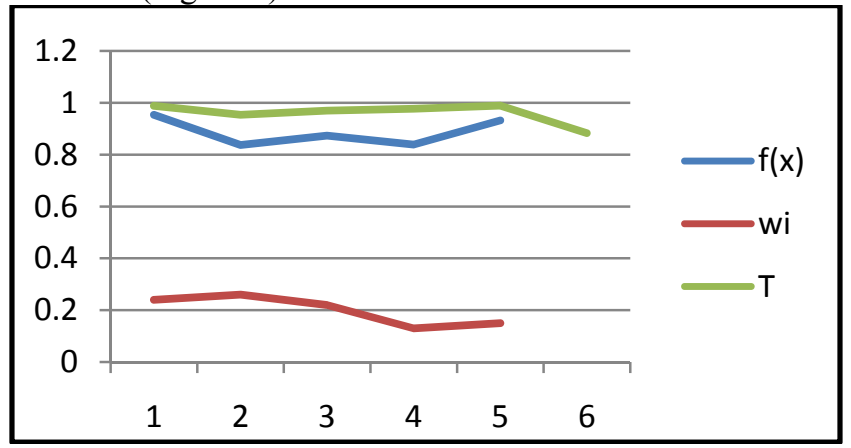

Figure 9: Quality of service during a handover between two levels.

$$
\mathrm{T}=0,883 \in[0,1]
$$

Interpretation of 5,7,8,9 figures.

We find, in the light of the two first figures, a quality improvement services in the second. We also find that when you change the aggregate function, the transmission rate of the fourth figure provides a better quality of service than the third. That confirms that the second and the fourth figure show some characteristics near reality; for it is rare that the flow rate is constant in a cell (when one moves away from the access point). 


\section{Discussion}

Discussed during our studies to propose a protocol to ensure seamless mobility in wireless community networks. Prior Mobile IP (MIP) [1,4] has been proposed to solve the communication problems of rupture during the movement of mobile nodes in IP networks, but [5] we do not understand that it supports fast handoff for applications sensitive delay and packet loss. To resolve this issue, MIP extensions, such as MIP fast transfer (FMIP, fast handover for MIP) and Hierarchical MIP (HMIP, hierarchical MIP), but they do not guarantee a period of minimal notes and a loss of tolerable packages. A second protocol to propose is SIP. In [4], SIP does not provide the transparent management of the handover.]. A third protocol SCTP / mSCTP proposed and thanks to its Multi-homing technique to open multiple IP connections to the same association, and error control mechanism to detect loss, rupture sequences or duplication of packets and dynamic configuration of addresses in an association. The Markov chain have enabled our modeling mSCTP and the aggregation function allowed us to show the quality of service.

\section{Conclusion}

It is question for us throughout this article to present the mobility management in own community networks in our communities (Universities, Institutes, School..). We have made studies on various mobility management protocols at the network level, application and transportation while presenting the advantages and disadvantages.

These studies led us to propose the SCTP / mSCTP as the promoter protocol of the mobility in wireless community networks, given its advantages presented through the technique of multihoming. the mSCTP mobility protocol is modeled by using the markov chains continuously. Finally researches carried out on aggregation functions show the quality of service on the audio type of application.

\section{References}

[1] Jean-Baptiste SANGLA. Résumé de l'étude SagaTel sur les marchés du WiFi en France(2007).

[2] Philippe ATELIN. Réseaux sans fil 802.11: Technologie - Déploiement - Sécurisation(2010),224pages

[3] Prescriptions de gestion de la mobilité au niveau des interfaces de nœuds de réseau pour les systèmes postérieurs aux IMT-2000, $(12 / 2004)$

[4] GUY PULLOLE. Les réseaux, $5^{\mathrm{eme}}$ et $6^{\mathrm{me}}$ édition septembre(2006)

[5] Mohamed Rabbie Naimi. Évaluation des Performances de Mobile SCTP dans 1'Equilibrage de Charge basée sur le Type de Flux dans les réseaux NEMO. Mémoire de Magister, UNIVERSITE ABOUBEKR BELKAID d' Algérie(2012), 117Pages.

[6] Nicolas Montavont. LA MOBILITE DANS LES RESEAUX IP( 2001).

[7] Eric Meurisse. L’UMTS et les hauts débits mobiles».( 2007).

[8] R. Stewart, Q. Xie, K. Morneault, C. Sharp, H. Schwarzbauer, T. Taylor, I. Rytina, M. Kalla, L.Zhang, V. Paxson, «Stream Control Transmission Protocol», RFC2960, Internet Engineering Task Force, (2000).

[9] J.Iyengar, K.Shah, P.Amer, R.Stewart, «Concurrent Multipath Transfer Using SCTP Multihoming», SPECTS (2004).

[10] M. Arif, S. Hafid, T.Brouard1 N. Vincent. AWFO (un opérateur d'agrégation) pour la reconnaissance des formes AWFO (an aggregation operator) for pattern recognition (2005).

[11] Gérard Michel Cochard. Réseaux cellulaires GSM ; GPRS;UMT( 2002).

[12] Jean-Luc Marichal. Fonctions d'agrégation pour la décision,4 (2003).

[13] RECOMMANDATION UIT-R M.1079-2,(1994-2000-2003).

[14] DAVID COUPIER.PROCESSUS STOCHASTIQUES, ouvrage ,janvier (2014),54pages.

[15] E. Pommiès, S. Robin. Introduction aux chaînes de Markov homogènes, ( 2004).

[16] Michel Grabisch, Patrice Perny. Agrégation Multicritère, 12 (3) (2002). 